\title{
Análise da eficácia de acaricidas sobre o carrapato Boophilus microplus, durante a última década, na região sul do Rio Grande do Sul
}

\author{
Acaricids efficacy analysis on Boophilus microplus tick, in the last decade in the southern of \\ Rio Grande do Sul
}

Nara Amélia Farias ${ }^{\mathrm{I}}$ Jerônimo Lopes Ruas ${ }^{\mathrm{II}}$ Tânia Regina Bettin dos Santos ${ }^{\mathrm{II}}$

\section{RESUMO}

A região sul do Rio Grande do Sul caracteriza-se por uma bovinocultura com predomínio de raças européias, tanto para produção de carne, quanto de leite. Essa região é considerada área marginal de ocorrência do carrapato Boophilus microplus, cujo parasitismo ocorre entre final de setembro e meados de maio devido às baixas temperaturas do inverno. No entanto, as perdas causadas por esse parasito são elevadas, o que tem sido agravadas pela seleção de populações de carrapatos resistentes aos acaricidas. O presente trabalho teve como objetivo estudar a situação de sensibilidadel resistência do carrapato bovino, assim como a dinâmica de uso dos diferentes grupos acaricidas disponíveis no mercado, na região sul do Rio Grande do Sul, na última década. Durante o período foram avaliadas amostras de carrapatos provenientes de 124 propriedades, de 14 municípios da região. Em cada propriedade foram coletadas teleóginas e informações relativas ao manejo. No Laboratório de Parasitologia do Instituto de Biologia da UFPel, foi realizado teste de imersão de teleóginas. Observou-se que durante o primeiro triênio os produtos mais utilizados eram à base de piretróide, determinando uma seleção de populações resistentes a esse princípio ativo. No terceiro triênio os produtos a base de amitraz dominaram o mercado, ocorrendo também seleção de populações resistentes. No primeiro triênio, todas as populações de carrapato analisadas eram sensíveis ao amitraz (índice de eficácia $=95 \%$ ), enquanto que, ao final do estudo, no terceiro triênio, esse índice de eficácia foi detectado em apenas $79 \%$ das populações estudadas. Foram detectadas falhas de manejo na região, como número de aplicação superior a seis por ano, aplicação de acaricida somente quando visualizadas as formas adultas e uso exclusivo de acaricidas para o controle do carrapato, que predispõem a seleção de populações resistentes.

Palavras-chave: controle de carrapatos, carrapaticidas, Boophilus microplus, bovinos.

\begin{abstract}
In the southern region of Rio Grande do Sul State, European cattle represent the most important stock used for meat and milk production. This region is considered a marginal area for the occurrence of the tick Boophilus microplus, which parasites the cattle between the end of September and early May. The damage caused by this parasite is high, which has been aggravated by the selection of tick populations resistant to acaricids. The aim of this present research was to evaluate the sensibility/resistance situation of Boophilus microplus, as well as the dynamic of the use of different acaricids in the last decade. During the period of study, tick samples from 124 farms, collected in 14 counties of the region, were analyzed. In each farm engorged female ticks and information about the acaricids management were collected. In order to test the sensibility/resistance we used the immersion test of the engorged females tick performed at the Laboratório de Parasitologia do Instituto de Biologia da UFPel. We observed that in the first three years of the last decade a selection of resistant tick populations was induced by pyretroids products. Products that used amitraz as a main compound, the most important acaricids used in the last three years of the decade, also induced resistant selection in the tick population to this drug. In the first three years of the studied period all tick populations analyzed where sensitive to amitraz (efficacy index $=95 \%$ ). However, in the last three years of the decade, the efficacy index detected drop to $79 \%$ in the studied populations. Managing inaccuracy in the region where detected such as, number of application higher than six times a year, application of acaricids only when the adults forms where visualized and the exclusive use of the acaricid for the tick control, that might predispose to the selection of resistant populations.
\end{abstract}

Key words: tick control, acaricides, Boophilus microplus, bovine.

IInstituto de Biologia, Departamento de Microbiologia e Parasitologia, Universidade Federal de Pelotas (UFPel), CP 354, Pelotas, RS, Brasil. E-mail: nafarias@ufpel.edu.br. Autor para correspondência.

IIFaculdade de Veterinária, UFPel, Pelotas, RS, Brasil. 


\section{INTRODUÇÃO}

Na bovinocultura da região sul do Estado do Rio Grande do Sul, predomina a exploração de raças européias, tanto para produção de carne, quanto para a produção de leite. A criação desses animais tem favorecido a proliferação do carrapato, pois, ao encontrar esses hospedeiros sensíveis, os carrapatos podem atingir sua capacidade reprodutiva máxima.

Essa região é considerada uma área marginal de ocorrência do carrapato, pois as baixas temperaturas verificadas no período de junho a setembro inibem a fase de vida livre do parasito (BRUM et al., 1985). A infestação do gado é reiniciada a partir de outubro, por larvas da progênie de teleóginas desprendidas no final do período favorável, que tiveram seus períodos de pré-postura, postura e eclosão muito prolongados (FARIAS et al., 1986).

A presença de hospedeiros sensíveis, somada às condições ambientais favoráveis, especialmente entre os meses de setembro até meados de maio, levam a um aumento da população de Boophilus microplus. Segundo GONZALES (2003), essa superpopulação de carrapatos, com forte pressão de carrapaticidas, leva a uma seleção de indivíduos resistentes às drogas.

O surgimento de populações de $\boldsymbol{B}$. microplus resistentes aos acaricidas vem sendo crescente em todas as regiões onde o parasito encontra condições favoráveis ao seu desenvolvimento, como na maioria dos países da América do Sul e da América Central, África do Sul e Austrália. No Brasil, existem populações resistentes aos diferentes acaricidas, em vários estados (FARIAS, 1994; MARTINS et al., 1995; FARIAS, 1999; FURLONG et al., 2004; FERNANDES et al., 2004).

Segundo FARIAS (1999), nos últimos anos, devido ao aumento da resistência aos piretróides, estes foram substituídos pelas formamidinas (amitraz), com os inconvenientes de ter baixa ação inseticida, não atuar em pH baixo e já existirem populações resistentes aos componentes amidínicos, que precisam ser controladas com a utilização de endectocidas e fluazuron. Em 2002, foi confirmada a resistência de uma população de $\boldsymbol{B}$. microplus ao amitraz, no Rio Grande do Sul (MILLER et al; 2003).

Esses estudos demonstram uma crescente resistência das populações de carrapato no Brasil aos diferentes grupos químicos. Trata-se de uma situação grave, uma vez que a utilização de químicos é indispensável na maioria dos planos de controle integrado e o custo para o desenvolvimento de novas moléculas pela indústria é cada vez mais alto, além de despender um tempo geralmente maior que o necessário para o surgimento da resistência (KEMP et al.,1998).

Para prevenir e retardar o desenvolvimento da resistência e reduzir seu impacto, são necessárias pesquisas que permitam conhecer a extensão do problema com rapidez e precisão, além de avaliar possíveis medidas alternativas (BAXTER et al., 1999).

O presente trabalho teve como objetivo estudar a situação de sensibilidade/resistência do carrapato bovino, aos diferentes grupos de acaricidas disponíveis no mercado e sua relação com a dinâmica de uso dessas drogas e com as características do controle do ácaro na região sul do Rio Grande do Sul, na última década.

\section{MATERIAL E MÉTODOS}

O estudo foi realizado entre março de 1997 e fevereiro de 2006 no Laboratório de Parasitologia, do Instituto de Biologia da Universidade Federal de Pelotas (UFPel), RS. Durante este período foram avaliados carrapatos, provenientes de 124 propriedades destinadas à bovinocultura de carne e/ou leite, localizadas em 19 diferentes municípios da região sul do Rio Grande do Sul (Pelotas, Pedro Osório, Cerrito, Morro Redondo, Capão do Leão, Camaquã, Cristal, São Lourenço do Sul, Canguçu, Piratini, Herval, Rio Grande, Hulha Negra, Rosário do Sul, Pinheiro Machado, Bagé, Lavras do Sul, Arroio Grande e Alegrete).

Em cada propriedade, foram coletadas teleóginas (fêmeas ingurgitadas) de vários bovinos que não tivessem recebido acaricida há, no mínimo, 20 dias. Foram coletadas informações relativas ao controle do carrapato na propriedade, tais como: produto utilizado, critério para a aplicação de acaricidas, tipos de aplicação, número médio de aplicações por ano, controle da mosca-dos-chifres (Haematobia irritans) e uso de alternativas para o controle do carrapato.

No Laboratório de Parasitologia foi realizado o teste de imersão de teleóginas, segundo DRUMMOND et al. (1973), a fim de determinar sua sensibilidade aos diferentes princípios ativos disponíveis no mercado. Grupos de 10 fêmeas foram pesados e imersos durante cinco minutos nos diferentes acaricidas, de acordo com a diluição recomendada pelo fabricante. Depois disso, 
os grupos foram incubados em estufa a $27^{\circ} \mathrm{C}$ e umidade relativa superior a $80 \%$. Ao final de duas semanas, foi avaliada a mortalidade e os ovos form pesados e incubados para, posteriormente, ser estimada a eclodibilidade. Sempre foi utilizado um grupo controle, imerso em água. O índice de eficácia de cada princípio foi determinado segundo a fórmula descrita por DRUMMOND et al. (1973).

Para observar as variações de uso e eficácia dos diferentes princípios ativos, durante os nove anos de estudo, os resultados foram agrupados em triênios: $1^{\circ}$ - Dados obtidos entre março de 1997 e fevereiro de 2000 (50 propriedades); ㄴo-Dados obtidos entre março de 2000 e fevereiro de 2003 (31 propriedades); $3^{\circ}$ Dados obtidos entre março de 2003 e fevereiro de 2006 (43 propriedades).

A análise estatística foi realizada com o auxílio do programa Statistix 8.0, sendo que, para os dados percentuais foi utilizado o teste que Quiquadrado e para comparação entre as médias o teste de Kruskal-Wallis.

\section{RESULTADOS E DISCUSSÃO}

O controle do carrapato é feito exclusivamente por meio da aplicação de acaricidas em $100 \%$ das propriedades analisadas. Isso ocorre somente quando a infestação dos animais é percebida à distância (formas adultas). Esse fator contribui para a seleção de populações resistentes, já que o tratamento acaricida é feito no momento em que há teleóginas, que são os ínstares mais resistentes (GONZALES, 2003).

Na região, o modo de aplicação de acaricida predominante é a imersão, diferente de outras regiões do país, como a Sudeste e o Centro-oeste, onde o método de aplicação mais utilizado é o de aspersão (ROCHA et al., 2006). Foi observado que nos últimos triênios houve uma tendência de utilizar mais a aplicação por aspersão e pour-on, facilitando o rodízio entre os princípios ativos (Tabela 1).
Tabela 1 - Número e freqüência (\%) das propriedades analisadas, nos diferentes triênios, segundo o tipo de aplicação do acaricida.

\begin{tabular}{|c|c|c|c|}
\hline \multirow{2}{*}{ Tipo de aplicação } & \multicolumn{3}{|c|}{---------------Triênio--------------- } \\
\hline & $1^{0}$ & $2^{\underline{0}}$ & $3^{0}$ \\
\hline Imersão & 41 (82\%) & 22 (71\%) & 30 (70\%) \\
\hline Aspersão & $5(10 \%)$ & $5(17 \%)$ & 7 (17\%) \\
\hline Pour-on & $4(8 \%)$ & $4(12 \%)$ & $6(13 \%)$ \\
\hline
\end{tabular}

O número de aplicações de carrapaticida por ano é igual ou superior a seis na maioria das propriedades estudadas, a exemplo do verificado por ROCHA et al. (2006). Essa média foi mantida durante todo o período experimental, o que, segundo NOLAN (1990) e VIVAS et al. (2006), constitui um fator de risco para seleção de populações resistentes.

O controle do carrapato na região era feito sobretudo com produtos a base de piretróides, que foram gradativamente sendo substituídos pelo amitraz, (Figura 1), o que já havia sido detectado por FARIAS (1999).

A dinâmica entre os princípios ativo devese à baixa eficácia dos piretróides sobre as populações estudadas, o que pode ser verificado na figura 2 . Um dos fatores determinantes foram as medidas de controle da $\boldsymbol{H}$. irritans (mosca-dos-chifres), com o uso destas moléculas em subdose e com maior freqüência. $\mathrm{O}$ presente estudo confirma essa hipótese, ao constatar que $86 \%$, 60\% e $69 \%$ dos proprietários analisados no $1^{\circ}$, $2^{\circ}$ e $3^{\circ}$ triênio, respectivamente, usam piretróides para controlar a mosca-dos-chifres em seus rebanhos.

A análise estatística, comparando o mesmo princípio ativo em cada triênio, demonstrou diferença significativa $(\mathrm{P}<0,05)$ entre os triênios para o amitraz $(\mathrm{P}<0,0113)$ e as associações de piretróides + organofosforados $(\mathrm{P}<0,0256)$, ocorrendo diminuição

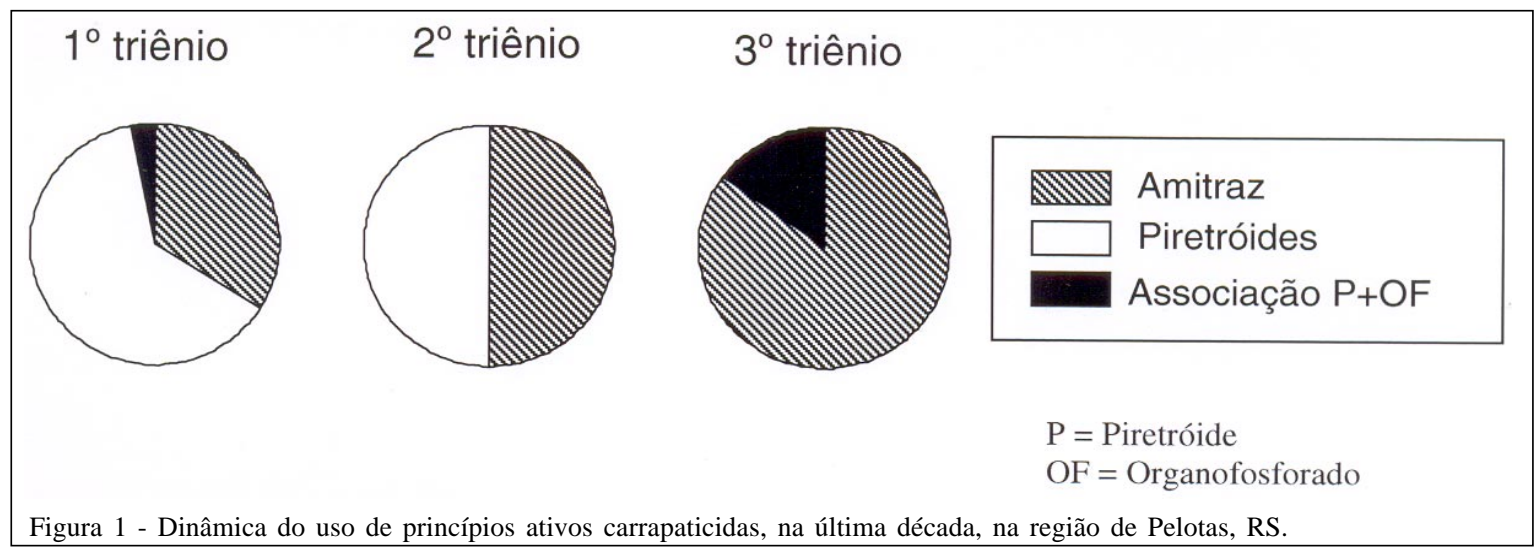

Ciência Rural, v.38, n.6, set, 2008. 


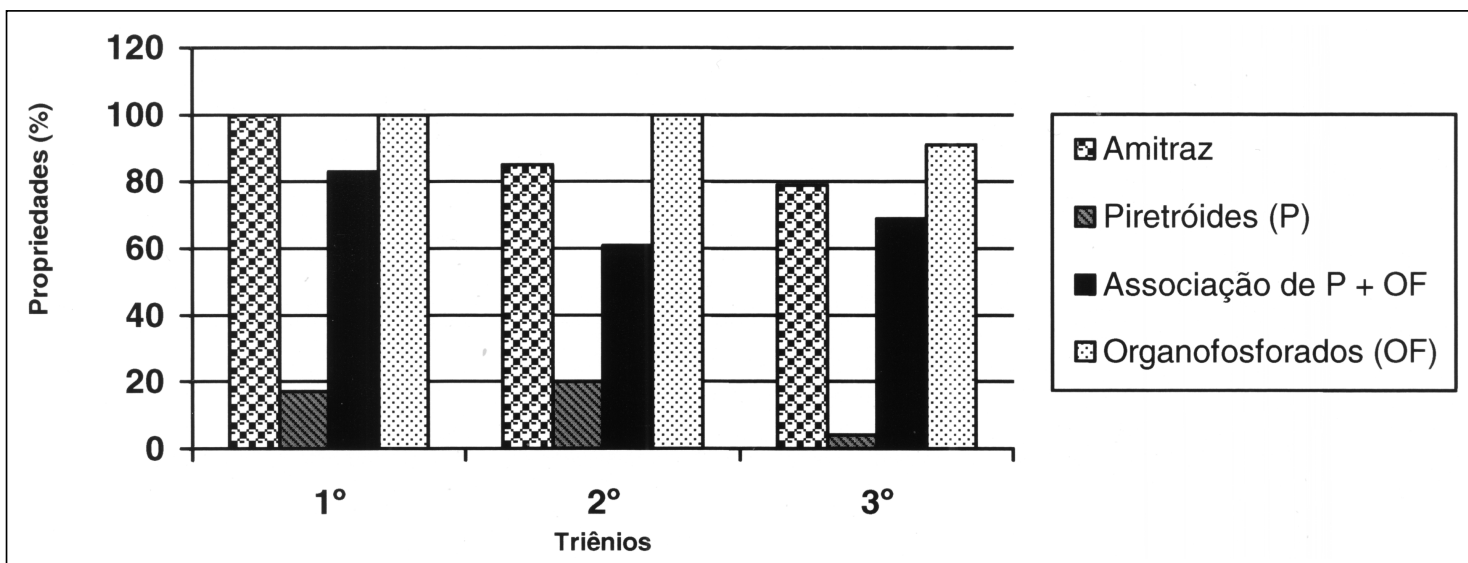

Figura 2 - Freqüência de propriedades onde o índice de eficácia dos acaridas sobre Boophilus microplus foi superior ou igual a 95\%, nos diferentes triênios do experimento, no sul do Rio Grande do Sul.

progressiva da eficácia destes produtos ao longo do período (Figura 2).

Deve ser considerado que houve uma inversão de princípios ativos utilizados, pois enquanto no começo do estudo os produtos mais usados eram a base de piretróides e no final do mesmo as formamidinas (amitraz) dominavam o mercado (Figura 1). O percentual de propriedades com eficácia do amitraz superior ou igual a $95 \%$ era de $100 \%$ no $1^{\circ}$ triênio e, com a intensificação de seu uso, houve seleção de populações resistentes, fazendo com que esse percentual decrescesse para $79 \%$ no $3^{\circ}$ triênio.

Ao serem comparadas as eficácias médias de cada princípio ativo, entre os três triênios, (Tabela 2), constata-se que a diferença entre elas não é significativa $(\mathrm{P}>0,05)$. No entanto, ficou demonstrado estatisticamente que o percentual de populações menos sensíveis ao amitraz e às associações de piretróides + organofosforados aumentou progressivamente no decorrer do experimento (Figura 2). Esse quadro é agravado pela grande comercialização de gado que ocorre atualmente, possibilitando a dispersão de populações de $\boldsymbol{B}$. microplus com diferentes graus de resistência às drogas.

\section{CONCLUSÃO}

A região estudada apresenta populações de $\boldsymbol{B}$. microplus com diferentes graus de sensibilidade aos princípios ativos, tendo como agravante falhas de manejo detectadas na maioria das propriedades. Essas falhas podem ser resumidas em uso exclusivo de acaricida para o controle do carrapato, aplicação dos produtos somente após a detecção de formas adultas, número de aplicações ser superior a seis por ano e controle inadequado de $\boldsymbol{H}$. irritans.

$\mathrm{O}$ aumento do uso de produtos à base de amitraz e de associações de piretróides + organofosforados favoreceu a seleção de carrapatos resistentes a essas moléculas no decorrer do tempo.

Fica evidente a necessidade de maior orientação de técnicos e produtores sobre o uso de alternativas para o controle do carrapato e aplicações estratégicas de carrapaticidas, o que prolongaria sua a vida útil, pois quanto maior a pressão acaricida mais rápida a seleção de populações resistentes.

Tabela 2 - Índice de eficácia in vitro de diferentes acaricidas sobre teleóginas de Boophilus microplus, na última década, na região sul do RS.

\begin{tabular}{|c|c|c|c|c|c|c|c|c|c|}
\hline \multirow{2}{*}{ Princípio Ativo } & \multicolumn{3}{|c|}{--------10 Triênio-------- } & \multicolumn{3}{|c|}{--------2o Triênio-------- } & \multicolumn{3}{|c|}{--------3o Triênio-------- } \\
\hline & Mínima & Máxima & Média \pm DP & Mínima & Máxima & Média $\pm \mathrm{DP}$ & Mínima & Máxima & Média $\pm \mathrm{DP}$ \\
\hline Amitraz & 96 & 100 & $100 \pm 0,70^{\mathrm{a}}$ & 0 & 100 & $90 \pm 25,60^{a}$ & 0 & 100 & $92 \pm 20,27^{\mathrm{a}}$ \\
\hline Piretróide & 0 & 100 & $55 \pm 34,38^{b}$ & 0 & 100 & $49 \pm 38,34^{b}$ & 0 & 100 & $42 \pm 30,04^{b}$ \\
\hline Organfosf + Piretróide & 75 & 100 & $96 \pm 5,12^{c}$ & 72 & 100 & $90 \pm 17,05^{c}$ & 20 & 100 & $93 \pm 16,42^{c}$ \\
\hline Organof. & 100 & 100 & $100 \pm 0,02^{\mathrm{d}}$ & 100 & 100 & $100 \pm 0,09^{d}$ & 88 & 100 & $99 \pm 3,30^{d}$ \\
\hline
\end{tabular}

Médias seguidas de letras iguais, na mesma linha, não diferem significativamente entre si (P>0,05) pelo teste de Kruskal-Wallis (Programa Statistix 8.0). 


\section{REFERÊNCIAS}

BAXTER, G.D. et al. Detecting resistance to organophosporados and carbamates in the cattle tick, Boophilus microplus, with a propoxur based chemical test. Experimental Applied Acarology, n.23, p.907-914, 1999.

BRUM, J.G.W et al. Postura e eclosão de Boophilus microplus em diferentes localizações geográficas do RS, Brasil. Arquivos Brasileiros de Medicina Veterinária e Zootecnia, v.37, n.6, p.581-587, 1985.

DRUMMOND, R.O. et al. Boophilus annulatus and Boophilus microplus. Laboratory tests of insecticides. Journal of Economic Entomology, n.66, p.130-133, 1973.

NOLAN, J. Acaricide resistence in the cattle tick Boophilus microplus. In: REPORT OF WORKSHOP LEADER - FAO/ UN consultant, Porto Alegre, RS, Brazil. Abstract... Porto Alegre, 1994. p.21-25.

FARIAS, N.A. Resistência do carrapato Boophilus microplus a acaricidas no noroeste do Estado de São Paulo. In: WORKSHOP - ACARICIDE RESISTANCE IN THE CATTLE TICK Boophilus microplus, 1994, Porto Alegre, RS, Brasil. Anais... Porto Alegre: FAO/UN, 1994. p.10-13.

FARIAS, N.A. et al. Carrapato bovino: distribuição populacional e correlação carga parasitária/hematócrito do hospedeiro. In: ENCONTRO DE PESQUISAS VETERINÁRIAS, 6., 1986, Pelotas, RS, UFPel. Anais... Pelotas: Faculdade de Veterinária, 1986 . p.23.

FARIAS, N.A. Situación de la resistencia de la garrapata Boophilus microplus em la región sur de Rio Grande Del Sur, Brazil. In: SEMINÁRIO INTERNACIONAL DE PARASITOLOGIA ANIMAL, 4., 1999, Puerto Vallarta, Mexico. Anais... Puerto Vallarta: CONASAGA, 1999. p.25-30.

FERNANDES, K. R. et al. Características do controle químico do Boophilus microplus no estado do Rio de Janeiro e a relação com a resistência a carrapaticidas. In: CONGRESSO BRASILEIRO DE PARASITOLOGIA VETERINÁRIA, 8., 2004,
Ouro Preto, MG, Brasil. Anais... Ouro Preto: Revista Brasileira de Parasitologia, $2004 . \quad$ p.307.

FURLONG, J. et al. Diagnóstico in vitro da sensibilidade do carrapato Boophilus microplus a acaricidas. In: CONGRESSO BRASILEIRO DE PARASITOLOGIA VETERINÁRIA, 8., 2004, Ouro Preto, MG, Brasil. Anais... Ouro Preto: Revista Brasileira de Parasitologia, 2004. p.305.

GONZALES, J.C. O controle do carrapato do boi. 3.ed. Passo Fundo: Universidade de Passo Fundo, 2003. 128p.

KEMP, D.H. et al. Acaricide resistence in the cattle ticks Boophilus microplus and Boophilus decoloratus. Roma : FAO, 1998. p.1-32. (Report to the Animal Health Services FAO).

MARTINS, J.R.S. A situation report on resistance to acaricides by the cattle tick Boophilus microplus in the state of Rio Grande do Sul, Southern Brazil. In: SEMINÁRIO INTERNACIONAL DE PARASITOLOGIA ANIMAL, 3., 1995, Acapulco, México. Anais... Acapulco: INIFAP, 1995. p.1-8.

MILLER, R.J. et al. Use of a modified-larval packet test (LPT) to measure amitraz susceptibility in Boophilus microplus in Brazil, New Caledonia, and Uruguay, and comparison of the modified - LPT to a modified-Shaw technique for amitraz testing in B. microplus. In: INTERNATIONAL SEMINAR IN ANIMAL PARASITOLOGY, 5., 2003, Merida,Yucatan, México. Anais... Mérida: INEGI, 2003. p.1-3.

NOLAN, I. Acaricide resistence in single and multi-host ticks and strategies for control. Parasitology, v.32, n.1, p.145153, 1990.

ROCHA, C.M.B.M. Percepção dos produtores de leite do município de Passos, MG sobre o carrapato Boophilus microplus (Acari: Ixodidae), 2001. Ciência Rural, v.36, n.4, p.1235-1242, 2006.

VIVAS, R.I.R. Prevalence and potential risk fators for organophosphate and pyrethoid resistance in Boophilus microplus ticks on cattle ranches from the State of Yucatan, México. Veterinary Parasitology, n.136, p.335-342, 2006. 